\title{
Anesthésie et analgésie locorégionale en chirurgie abdominale et périnéale
}

\author{
Sous la direction de Marc Beaussier et Patrick Niccolai: Édition Arnette - Wolters \\ Kluwer France, 2010, 296 pages. ISBN 978-2-7184-1228-3
}

\author{
Pierre Drolet, MD
}

Received: 20 January 2011/ Accepted: 26 January 2011/Published online: 8 February 2011

(C) Canadian Anesthesiologists' Society 2011

Les blocs neuraxiaux ont longtemps constitué l'essentiel de l'arsenal anesthésique en matière d'analgésie et d'anesthésie de l'abdomen et du périnée. Encore aujourd'hui, la rachianesthésie et la péridurale demeurent des techniques universellement reconnues et auxquelles les auteurs d'Anesthésie et analgésie locorégionale en chirurgie abdominale et périnéale consacrent des chapitres visant à actualiser nos connaissances. Bien que pertinents, ces chapitres ne représentent toutefois qu'une partie relativement mineure de l'ouvrage rédigé sous la direction de Beaussier et Niccolaï. En effet, Anesthésie et analgésie locorégionale en chirurgie abdominale et périnéale se veut avant tout une mise à jour des techniques aptes à être substituées aux blocs neuraxiaux lorsque ceux-ci sont contre-indiqués. Les développements récents en matière de thromboprophylaxie, de chirurgie ambulatoire et de coelioscopie ont quelque peu réduit la place des blocs neuraxiaux. En effet, la péridurale est parfois devenue trop risquée ou trop lourde face à la réduction des durées d'hospitalisation et de l'agression chirurgicale. C'est donc dans cette optique que les auteurs de cet ouvrage proposent une série de chapitres consacrés aux diverses approches locorégionales possibles afin de procurer une analgésie de qualité, de manière relativement simple et dénuée de la majorité des inconvénients associés à l'injection péridurale continue.

Signe des temps, cet ouvrage fait une place importante aux techniques échoguidées, lesquelles ont contribué à l'engouement récent pour l'anesthésie locorégionale. Notons d'emblée qu'Anesthésie et analgésie locorégionale en chirurgie abdominale et périnéale représente plus qu'un

P. Drolet, MD ( $\square)$

Université de Montréal, Montréal, QC, Canada

e-mail: Courriel.pdrolet@aei.ca simple référentiel de blocs. Ainsi, l'ouvrage se distingue de plusieurs parutions récentes qui n'ont pour seul priorité que l'aspect technique des approches proposées. Plusieurs sections font état de considérations (bénéfices, limitations, impacts postopératoires, etc.) qui vont bien au-delà de la simple description technique. On retrouve même un chapitre entier consacré à la réhabilitation et l'impact que peut avoir le contrôle de la douleur. Une telle approche donne à l'ouvrage un caractère exhaustif qui plaira à plusieurs, mais qui risque de rebuter quelque peu celui qui a pour seule ambition d'obtenir rapidement une description succincte d'un bloc particulier. Le volume propose un éventail de techniques et le lecteur avide d'information trouvera des descriptions anatomiques adaptées aux approches suggérées. Les techniques échoguidées ne sont pas les seules à y être décrites. Les voies d'abord reposant sur la neurostimulation ou la perte de résistance sont aussi présentées, lorsqu'elles sont possibles. De nouveau, une telle abondance d'information plaira à certains mais risque, à l'occasion, d'être perçue comme encombrante.

Les auteurs ayant contribué à cet ouvrage, dont plusieurs sont des experts internationaux en matière d'anesthésie locorégionale, nous présentent une écriture soignée et un choix judicieux d'images abondantes, variées, généralement claires et pertinentes, surtout en ce qui touche la réalisation des techniques échoguidées. De manière plus précise, Anesthésie et analgésie locorégionale en chirurgie abdominale et périnéale contient des chapitres portant sur l'utilisation du TAP (transversus abdominis plane) bloc, du bloc combiné ilio-inguinal et ilio-hypogastrique, et du bloc des grands droits. Il s'agit là de techniques avec lesquelles les cliniciens férus d'anesthésie locorégionale sont souvent familiers. De nouveau, je souligne le caractère exhaustif de ces textes. Un chapitre consacré spécifiquement à l'apport de l'anesthésie locorégionale en chirurgie périnéale fait état 
de l'utilisation du bloc pénien, une technique connue d'une forte proportion d'anesthésiologistes canadiens. On y retrouve aussi le bloc du cordon spermatique, le bloc périprostatique et l'infiltration pour pose de prothèse sous-urétrale, techniques avec lesquelles la majorité d'entre nous sont peu familiers. Le bloc paracervical et le bloc pudental, des techniques généralement utilisées au Canada par nos collègues chirurgiens ou gynécologues, font aussi l'objet de descriptions détaillées. Deux chapitres portent respectivement sur l'instillation intrapéritonéale à visée analgésique et l'infiltration cicatricielle, cette dernière pouvant être réalisée en injection unique ou perfusion continue. On retrouve aussi des sections consacrées à la prise en charge des patients ambulatoires et à la gestion des douleurs chroniques.

Le contenu du volume est donc riche et de qualité, mais le lecteur qui souhaite y retrouver l'ensemble des informations relatives à une technique précise doit être prêt à consulter plus d'un chapitre. La taxonomie adoptée par les éditeurs favorise un certain morcellement de l'information, laquelle fait aussi l'objet de répétitions. Par exemple, l'inclusion d'un chapitre portant spécifiquement sur l'innervation abdominopelvienne et d'un autre présentant une vue d'ensemble du repérage échographique pour les blocs abdominopérinéaux est intéressante, mais plusieurs des éléments qu'on y trouve sont répétés de manière partielle dans les chapitres consacrés spécifiquement aux diverses approches. Le lecteur désireux d'avoir accès aux informations, et surtout aux images les plus intéressantes contenues dans l'ouvrage, ne doit donc pas hésiter à consulter plus d'une section. Dans l'ensemble, Anesthésie et analgésie locorégionale en chirurgie abdominale et périnéale est un ouvrage de référence contenant une foule de renseignements pertinents. Il plaira aux adeptes sérieux d'anesthésie locorégionale. Les cliniciens dont l'ambition unique est de profiter d'un maximum d'informations techniques et visuelles relatives à un bloc précis y trouveront aussi les éléments recherchés. Ils risquent cependant d'avoir à consulter plus d'un passage afin d'y arriver. 